\title{
Metabolomics Based Mechanism Exploration of Pulmonary Arterial Hypertension pathogenesis: Novel Lessons from Explanted Lungs
}

\section{Jingjing Ding}

Nanjing Medical University

Chunyan Chu

Nanjing Medical University

Zhengsheng Mao

Nanjing Medical University

Jiawen Yang

Nanjing Medical University

Jie Wang

Nanjing Medical University

Li Hu

Nanjing Medical University

Peng Chen

Nanjing Medical University

Yue Cao

Nanjing Medical University

Yan Li

Nanjing Medical University

Hua Wan

Nanjing Medical University

Dong Wei

Nanjing Medical University Affiliated Wuxi People's Hospital: Wuxi People's Hospital Jingyu Chen

Nanjing Medical University Affiliated Wuxi People's Hospital: Wuxi People's Hospital

Feng Chen

Nanjing Medical University

Youjia Yu ( $\nabla$ yuyoujia@njmu.edu.cn )

Nanjing Medical University https://orcid.org/0000-0001-6050-7637 
Keywords: $\mathrm{PAH}$, lung transplantation, metabolomics, AKT pathway, prognosis

Posted Date: September 7th, 2021

DOI: https://doi.org/10.21203/rs.3.rs-820723/v1

License: (c) (1) This work is licensed under a Creative Commons Attribution 4.0 International License. Read Full License

Version of Record: A version of this preprint was published at Hypertension Research on March 30th, 2022. See the published version at https://doi.org/10.1038/s41440-022-00898-0. 


\section{Abstract}

Background: Metabolic pathways have been shown to participate in the pathogenesis of pulmonary arterial hypertension (PAH). We investigated the metabolic profile shifts to reveal molecular mechanisms underlying PAH.

Methods: Explanted human lung tissues from 18 PAH patients were collected. The lung tissues far from the tumor from 16 lung cancer patients were taken as controls. Lung tissues were analyzed by LC-MS/MS based non-target metabolomics method. Pathway analysis was performed with KEGG database and MetaboAnalyst 5.0. Statistical analysis including partial least squares discriminant analysis (PLS-DA), Student's t-test, Pearson's correlation, Chi-square test and Fisher's exact probability test were used. COX survival analysis model was applied to evaluate the predictive value of metabolites on prognosis. Protein expression levels were detected by Western blotting in human PAH lung tissues, rat monocrotaline-PAH lungs and hypoxia exposed human pulmonary artery smooth muscle cells (HPASMCs) to study the molecular mechanisms.

Results: Significant differences in metabolites and metabolic pathways were identified among PAH subgroups and control tissues. Spermine levels were positively correlated with the patients' cardiac outputs (COs). Levels of (2e)-2,5-dichloro-4-oxo-2-hexenedioic acid were positively correlated with the patient's serum creatinine (Scr) levels. Patients with higher levels of thymine had a better prognosis. Moreover, 7 differential metabolites were associated with AKT pathway. AKT pathway inactivation was confirmed in human and rat PAH lungs and hypoxia exposed HPASMCs.

Conclusions: Our findings provide the first metabolomics evidence for $\mathrm{PAH}$ pathogenesis by human lungs and may contribute to the improvement of therapeutic strategy.

\section{Background}

Pulmonary hypertension $(\mathrm{PH})$, known as a substantial global health issue with poor treatment and prognosis[1, 2], is defined by a mean pulmonary arterial pressure (mPAP) above $20 \mathrm{mmHg}$ at rest with a normal pulmonary artery wedge pressure (PAWP) $\leqslant 15 \mathrm{mmHg}$ and elevated pulmonary vessel resistance $(P V R) \geqslant 3$ Wood Units (WU)[3]. The prevalence of $\mathrm{PH}$ in general population is about $15-50$ cases per million individuals [4]. In the 6th World Symposium on Pulmonary Hypertension (WSPH), PH is classified into 5 groups based on pathophysiology and clinical presentation as follows: (1) pulmonary arterial hypertension (PAH), (2) PH due to left heart disease, (3) PH due to lung disease and/or hypoxia, (4) PH due to pulmonary artery obstructions, and (5) PH due to unclear and/or multifactorial mechanisms. As shown by data from most PAH registries in the USA and Europe, idiopathic PAH (iPAH) accounts for about $50-60 \%$ of all cases, followed by PAH associated with connective tissue disease and PAH associated with congenital heart disease (CHD-PAH). In Asia, the most common type is CHD-PAH (35.8\%), followed by iPAH (29.7\%) dominated by young and middle-aged women [5, 6]. And the 5-year overall survival rate of patients with $\mathrm{PAH}$ is only $59 \%$. Many PAH patients with right ventricular failure die within 
2 to 3 years after diagnosis if left untreated and are burdened with a significant cost of health-care. Though PAH has led to global healthy and social problems, the pathogenic mechanism of PAH has not yet been elucidated $[7,8]$.

Heightened proliferation of pulmonary arterial smooth muscle cells (PASMCs) leading to the thickening of vascular wall and narrowing of luman of pulmonary arteries is the most significant pathological feature of PAH. Recently, more investigators pay their attentions to metabolic pathways underlying PAH pathogenesis. The 'multi-omics profiles' on PASMC lines, hypoxic rodent models or peripheral blood from PAH patients, have made significant progresses to capture small changes in gene and protein expression $[2,5,9,10]$. A growing body of evidence shows that pathological process of PAH is accompanied by mitochondrial dysfunction, the interruption of glycolysis, the increase in fatty acid metabolism and changes in oxidation pathways[11], and partially explains the observed increase in PASMC proliferation and apoptosis resistance in PAH [12]. However, most of these studies were performed in animal tissue, to understand the metabolic changes in PAH human tissues is urgently needed but remain challenging.

Nowadays, lung transplantation was introduced to treat PAH patients[13]. Explanted lung tissues from PAH patients are the most direct and valuable samples for studying metabolic changes of PAH. Here, we performed a liquid chromatography-tandem mass spectrometry (LC-MS/MS)-based non-targeted metabolomics investigation in the explanted lung tissues from PAH patients. As far as we know, this is the first metabolomics study using human lung tissues from PAH patients. We provide direct evidences of the metabolic heterogeneity in lungs from severe PAH patients. Our results reveal novel differential metabolites and metabolic pathways in PAH, including several previously reported human plasma metabolites $[14,15]$, to outcrop new pathogenic mechanisms of PAH. What's more, AKT pathway was identified as the central signaling pathway associated with the metabolic network, which might be the possible internal mechanism for PAH pathogenesis.

\section{Methods}

\section{Human lung samples}

Lung samples were obtained from pulmonary arterial hypertension (PAH) patients receiving lung transplantation or lung cancer patients receiving pneumonectomys. And samples involved in this study were collected by surgical teams at the Department of Cardiothoracic Surgery in Wuxi People's Hospital. Protocols and informed consent forms for this study were approved by appropriate institutional review boards. Both of PAH and lung cancer were diagnosed according to the published consensus statements through combining clinical history, radiological finding, and pathological examination. Medical records of all participants were collected.

PAH patients met the inclusion and exclusion criteria were recruited as subjects. Patients were diagnosed with PAH by right heart catheterization (mPAP $>25 \mathrm{mmHg}$, PAWP $\leq 15 \mathrm{mmHg}$ and PVR $>3.0$ Wood units). PAH subtypes were classified by guidelines of the 6th World Symposium on Pulmonary Hypertension. 
Exclusion criteria were as follows: 1) severe pulmonary function impairment; 2) emergent heart transplant; 3) patients without enough data in the hemodynamic study. And the control samples came from lung cancer patients who underwent pneumonectomy in the same hospital. Patients with 1) serious or uncontrolled chronic diseases such as severe cerebrovascular disease or malignant tumour; 2) chronic severe lung disease (such as chronic obstructive pulmonary disease (COPD)); or 3 ) chronic progressive nephropathy were excluded.

All participants in this study agreed to donate their cut-off tissues for research. Written informed consents were obtained from all patients in accordance with the declaration of Helsinki. This study was approved by the Nanjing Medical University Ethics Committee ([2015] No.35, China).

\section{Sample collection and storage}

Samples of the lung tissues containing mostly small arteries to microarteries, where the most typical pathological change of PAH occurred, were collected from the explanted lungs during the surgery. The control samples came from lung tissues far from the tumor from lung cancer patients who underwent pneumonectomy. All samples were stored at $-80^{\circ} \mathrm{C}$.

\section{Histology of Lung Tissues}

Lung tissues were fixed in 4\% paraformaldehyde (PFA), embedded in paraffin and then sliced into $5-\mu m$ thick sections. Xylene and a graded series of ethanol (100\%, 90\%, $80 \%$ and $70 \%)$ were used for processing the sections. Sections were stained with hematoxylin and eosin (H\&E), and were analyzed under the microscopy (BX53, Olympus, Japan). To quantify pulmonary artery remodeling, 10 muscular arteries with diameters ranging from $20 \mu \mathrm{m}$ to $100 \mu \mathrm{m}$ from each group were randomly selected. Quantification data were shown as the ratio of medial thickness to cross sectional area (media/CSA)[16].

\section{Metabolites extraction}

The samples were thawed slowly at $4{ }^{\circ} \mathrm{C}$. Then, $25 \mathrm{mg}$ of each tissue was weighed and put into a $1.5 \mathrm{~mL}$ Eppendorf tube, and was added with $800 \mu \mathrm{L}$ extraction solution (Methanol: acetonitrile: water $=2: 2: 1, \mathrm{v}$ : v: v, pre-cooled at $\left.-20^{\circ} \mathrm{C}\right), 10 \mu \mathrm{L}$ internal standard sample $1,10 \mu \mathrm{L}$ internal standard sample 2 , and two small steel balls. They were grinded $(50 \mathrm{~Hz}, 5 \mathrm{~min})$ and sonicated in a water bath at $4^{\circ} \mathrm{C}$ for $10 \mathrm{~min}$. They were centrifuged at $25,000 \mathrm{rcf}$ for $15 \mathrm{~min}$ at $4^{\circ} \mathrm{C}$. And then, $600 \mu \mathrm{L}$ of the supernatant were taken, placed in a freeze vacuum concentrator, and dried. A complex solution $\left(200 \mu \mathrm{L}\right.$, methanol: $\mathrm{H}_{2} \mathrm{O}=1: \mathrm{g}$, v: v) was used to reconstitute. Samples were vortexed for $1 \mathrm{~min}$, sonicated at $4^{\circ} \mathrm{C}$ for $10 \mathrm{~min}$ and centrifuged with $25,000 \mathrm{rcf}$ for $15 \mathrm{~min}$ at $4^{\circ} \mathrm{C}$. At last, $20 \mu \mathrm{L}$ of the supernatant was taken from each sample, and was mixed with quality control (QC) samples to evaluate the repeatability and stability of the LC-MS analysis process.

\section{LCMS/MSbased metabolomics analysis}

Waters 2D UPLC (Waters, USA) tandem Q Exactive HF high-resolution mass spectrometer (Thermo Fisher Scientific, USA) was used for metabolite isolation and detection. 


\section{Chromatographic conditions}

The BEH C18 column was used as a chromatographic column $(1.7 \mu \mathrm{m}$ of column particle size, $2.1 \times 100$ $\mathrm{mm}$ of column size) (Waters). The positive mode mobile phase consists of $0.1 \%$ formic acid aqueous solution (Phase A) and methanol (Phase B). The negative mode mobile phase comprises of $10 \mathrm{mM}$ ammonium formate aqueous solution (Phase $\mathrm{A}$ ) and $10 \mathrm{mM}$ ammonium formate methanol solution (Phase $B$ ). The following gradients were used for elution: $0 \sim 1 \mathrm{~min}, 2 \%$ phase $B ; 1 \sim 9 \mathrm{~min}, 2 \%$ 98\% phase $B ; 9 \sim 12$ min, $98 \%$ phase $B ; 12 \sim 12.1$ min, $98 \%$ 2\% phase $B ; 12.1 \sim 15$ minutes, $2 \%$ Phase $B$. The flow rate was $0.35 \mathrm{~mL} / \mathrm{min}$ with the column temperature of $45^{\circ} \mathrm{C}$ and the injection volume of $5 \mu \mathrm{L}$.

\section{Mass spectrometry conditions}

A Q Exactive HF mass spectrometer (Thermo Fisher Scientific) was used for primary and secondary mass spectrometry data acquisition. Mass spectrometry scanning mass-to-nuclear ratio range was $70 \sim 1,050$, with 120,000 first-level resolution rate, $3 \mathrm{e} 6$ for AGC, and $100 \mathrm{~ms}$ as maximum injection time (IT). According to the precursor ion intensity, Top3 were selected for further fragmentation and secondary level signal information were collected. The secondary resolution rate was 30,000 and AGC was 1 e5, with 50 ms as the maximum IT, while the stepped normalized collision energies (NCE) was set at: 20, 40 and 60 eV.

Ion source (ESI) parameters were set as following: Sheath gas flow rate at 40 arbitrary units (arb), Aux gas flow rate at $10 \mathrm{arb}$, Spray voltage $(|\mathrm{KV}|)$ at 3.80 in positive ion mode, 3.20 in negative ion mode, the ion transfer tube temperature (Capillary temperature) at $320^{\circ} \mathrm{C}$, and the auxiliary gas heating temperature (Aux gas heater temp) at $350^{\circ} \mathrm{C}$.

To provide more reliable experimental results, samples were randomly sorted to reduce systematic errors. Every 10 samples were interspersed with one QC sample.

\section{Data processing}

\section{Data preprocessing}

Raw data collected via LC-MS / MS were imported into Compound Discoverer 3.0 (Thermo Fisher Scientific, USA) for data processing, which included: peak extraction, retention time correction within and between groups, combined ion addition, missing value filling, background peak labeling and metabolite identification. And finally compound molecular weight, retention time, peak area, identification results and other information were recorded. The metabolites were identified using a combination of BGI Library (HuaDa's self-built standard library), mzCloud and ChemSpider (HMDB, KEGG, LipidMaps).

The results exported by Compound Discoverer 3.0 were imported into metaX for data preprocessing, which mainly included: 1) probabilistic quotient normalization (PQN) to normalize the data for obtaining the relative peak area; 2 ) quality control-based robust LOESS signal correction (QC-RLSC) to correct the 
batch effect; 3) the coefficient of variations (CVs) of the relative peak area in all QC samples, which were bigger than $30 \%$ of compounds, was deleted.

\section{Data quality control}

Data quality was assessed by repeatability of QC sample detection. Contents included chromatogram overlays of QC samples, PCA, peak extraction difference in number and peak response intensity.

\section{Statistical analysis and screening of differential metabolites}

Partial least squares discriminant analysis (PLS-DA), and Student's t-test were used to identify the difference between groups and screen the metabolites. The screening conditions of differential metabolites were: 1 ) fold change $\geq 1.2$ or $\leq 0.83$; 2 ) $P<0.05$. If the sample number of a group was above 6 , variable important for the projection (VIP) $\geq 1$ would be an extra screening condition. Log 2 conversion was carried out for data.

A hierarchical cluster was used in the clustering algorithm, and Euclidean distance was used in distance calculation. Based on KEGG database (https://www.kegg.jp/), metabolic pathway enrichment analysis was carried out. Metabolic pathway analysis was performed using MetaboAnalyst 5.0. The pathway with $P$-value $<0.05$ was considered to be a differential metabolic pathway.

Pearson's correlation was used to analyze the relationship between differential metabolites and between differential metabolites and clinical indicators. Chi-square test and Fisher exact probability were used to test the difference of composition ratio between different groups. The predictive value of levels of differential metabolites on patient prognosis was evaluated by COX survival analysis model. $P<0.05$ was considered as statistical significance.

\section{Monocrotaline-induced PAH animal model}

This study was approved by the Nanjing Medical University Ethics Committee (IACUC-2105005, China).

The animals were maintained in a constant environmental condition (temperature $23 \pm 2^{\circ} \mathrm{C}$, humidity $55 \pm$ $5 \%, 12: 12 \mathrm{~h} \mathrm{light/dark}$ cycle) in the Animal Research Center of Nanjing Medical University. They had free access to food and water before and after all procedures.

Male Sprague Dawley rats ( $250 \mathrm{~g} ; \sim 7$ weeks old) received a single subcutaneous injection of normal saline (control, $n=6)$ or monocrotaline (MCT) (Sigma-Aldrich, USA) $(60 \mathrm{mg} / \mathrm{kg})(\mathrm{n}=6)$. After 28 days, both the MCT rats and the age-matched control rats were sacrificed for further analysis.

\section{Cell culture and treatment}

The PASMC line was purchased from Lonza (Swiss) and cultured in SmGM-2 BulletKit media (Lonza) containing $5 \%$ fetal bovine serum (FBS), growth factors, and antibiotics. Cells were incubated under $5 \%$ $\mathrm{CO}_{2}$ at $37^{\circ} \mathrm{C}$. 
PASMCs were exposed to hypoxia $\left(1 \% \mathrm{O}_{2}, 5 \% \mathrm{CO}_{2}\right.$, and $\left.1 \% \mathrm{FBS}\right)$ for $0,0.5,1,2,4,8,12$ and $24 \mathrm{~h}$. The initial number of cells in each group was kept the same.

\section{Western blotting analysis}

Proteins from cells were extracted by RIPA buffer containing PMSF (1 mM) (Beyotime, China) and were centrifuged at $4^{\circ} \mathrm{C}$ for $15 \mathrm{~min}$ at $12,000 \mathrm{rpm}$. The supernatants were quantified with the BCA protein assay kit (Beyotime). Equal amounts of total protein were separated by SDS-PAGE and transferred to PVDF membranes (Millipore, USA). Membranes were blocked for $1 \mathrm{~h}$ at room temperature with $5 \%$ skim milk and then incubated with the primary antibodies (rabbit-anti-AKT (CST, USA, 1:1000 dilution), rabbitanti-Phospho-AKT (Thr308) (CST, 1:1000 dilution), mouse-anti- $\beta$-actin (CST, 1:5000 dilution),mouse-antiGapdh (CST, 1:1000 dilution)) at $4^{\circ} \mathrm{C}$ overnight. After washing with TBST (Tris-buffered saline, $0.1 \%$ Tween-20), the membranes were incubated with second antibodies for $1 \mathrm{~h}$ at room temperature. Protein bands were visualized with the Enhanced Chemiluminescence Detection Kit (Thermo Fisher Scientific, USA) via the luminescent imaging system (Tanon, China). Image $\mathrm{J}$ was used to analyze the quantity of the protein bands.

\section{Results}

\section{Participants characteristics}

Pulmonary vascular walls in PAH patients were thicker than that in control patients (Fig. 1A, B). The PAH patients involved more females $(72.2 \%, 13 / 18)$ than the control patients $(25 \%, 4 / 16, P=0.02)$, and the PAH patients consisted of iPAH patients $(72.2 \%, 13 / 18)$ and CHD-PAH patients $(27.8 \%, 5 / 18)($ Table 1$)$.

Table 1

Clinical characteristics of the studied groups.

\begin{tabular}{|lllll|}
\hline & Control(n=16) & PAH(n=18) & t & $P$ \\
\hline Gender(F/M) & $12 / 4$ & $5 / 13$ & - & $0.02^{*}$ \\
\hline Age & $59.15 \pm 8.04$ & $30.44 \pm 9.68$ & 9.33 & $<0.001$ \\
\hline BMI & $23.42 \pm 3.38$ & $20.07 \pm 2.12$ & 3.2 & 0.003 \\
\hline PAH catology & & & & \\
\hline IPAH & - & 13 & & \\
\hline CHD-PAH & - & 5 & & \\
\hline *Fisher exact rate & & & \\
\hline
\end{tabular}

When comparing between iPAH patients and CHD-PAH patients, no significant differences in age, body mass index (BMI), and the hemodynamics of pulmonary arterial (PA) (functional capacity (FC), PA, mPAP, 
and ejection fractions (EF)) were observed (Table S2). However, CHD-PAH patients were all females that led to an unbalanced gender ratio between groups $(P<0.05)$.

\section{The metabolomics features of PAH lungs}

After data processing, 2,177 metabolites (1,903 in positive ion mode, 274 in negative ion model) were identified. Meanwhile, 1,787 (positive ion mode) and 266 (negative ion mode) metabolites were obtained by the coefficient of variations $(\mathrm{CVs}) \leq 30 \%$ as the further screening standard.

The PLS-DA suggested considerable differences of metabolites among $\mathrm{PAH}, \mathrm{CHD}-\mathrm{PAH}$ and control lung tissues (PLS-DA1 $=14.94 \%$, PLS-DA2 $=11.37 \%$ ) (Fig. 1C). A total of 281 significantly changed metabolites from iPAH vs. Control, CHD-PAH vs. Control and iPAH vs. CHD-PAH comparisons were found $(P<0.05)$ (Fig. 2A). Among them, glyceryl 1-(5-hydroxydecanoate), (4s)-4-[(2e)-2-octenoyloxy]-4(trimethylammonio) butanoate and 12-hydroxydodecanoic acid were identified as significantly differential metabolites in all 3 comparison groups. And data of the obviously changed metabolites between PAH and control lung tissues (VIP $\geq 1$, Fold change $\geq 1.50$ or $\leq 0.67, q<0.05)$ were listed in Table 2. 
Table 2

Difference in metabolism between patients and the normal

\begin{tabular}{|c|c|c|c|c|c|}
\hline Metabolite & $\begin{array}{l}\text { Fold } \\
\text { Change }\end{array}$ & VIP & $\begin{array}{l}\text { p Value } \\
(\mathrm{BH})\end{array}$ & $\begin{array}{l}\text { Direction of } \\
\text { Association }\end{array}$ & \\
\hline D-erythrose 4-phosphate & 0.063 & 1.5119 & 0.0253 & negative & down \\
\hline $\mathrm{N}$-acetyl-I-aspartic acid & 1.7203 & 1.0194 & 0.0236 & negative & up \\
\hline Neopterin & 0.0279 & 2.8146 & 0 & negative & down \\
\hline Sulfuric acid & 0.1028 & 1.5331 & 0.0126 & negative & down \\
\hline Spermidine & 0.3532 & 1.2085 & 0.0126 & positive & down \\
\hline Spermine & 0.1649 & 1.4819 & 0.0153 & positive & down \\
\hline D-ornithine & 0.6042 & 1.0338 & 0.0123 & positive & down \\
\hline $\begin{array}{l}\text { (2e)-2,5-dichloro-4-oxo-2- } \\
\text { hexenedioic acid }\end{array}$ & 1.976 & 1.1464 & 0.0012 & positive & up \\
\hline $\mathrm{N}$-acetyl-I-alanine & 0.5969 & 1.0318 & 0.0056 & positive & down \\
\hline Acetylcholine & 0.5697 & 1.1437 & 0.0002 & positive & down \\
\hline Thymine & 0.2311 & 1.8459 & 0.0007 & positive & down \\
\hline D-mannose 6-phosphate & 0.6155 & 1.0592 & 0.0003 & positive & down \\
\hline 4-pyridoxate & 0.4931 & 1.1831 & 0.005 & positive & down \\
\hline $\begin{array}{l}\text { 4-(2-aminophenyl)-2,4- } \\
\text { dioxobutanoic acid }\end{array}$ & 0.1607 & 1.4504 & 0.0425 & positive & down \\
\hline Aspartame & 0.5195 & 1.019 & 0.0288 & positive & down \\
\hline Dihydrouracil & 3.8225 & 1.3712 & 0.0046 & positive & up \\
\hline 24-hydroxycalcitriol & 7.7492 & 1.49 & 0.0065 & positive & up \\
\hline Gamma-linolenic acid & 0.4586 & 1.1153 & 0.0328 & positive & down \\
\hline
\end{tabular}

\section{Metabolic pathway changes in PAH lungs}

Metabolic pathway enrichment analysis was performed based on the KEGG database (Fig. 2B). By comparing between all PAH patients and control patients, 31 pathways were validated $(P<0.05)$ (Table S1), among which metabolic pathway was of the highest significance (Figure S1), where the significantly altered molecules with top three actual ratios were spermidine, spermine and D-ornithine. In more detail, glycerophospholipid metabolism, arginine and proline metabolism, glutathione metabolism, and alanine, aspartate and glutamate metabolism in iPAH vs. control, beta-alanine metabolism in CHD-PAH vs. 
control, and lysine degradation in iPAH vs. CHD-PAH comparisons were validated to be the significantly differential pathways $(P<0.05)$ (Fig. 2C-E).

\section{The relationships between metabolites and clinical features}

Analyzed by Pearson's correlation analysis, spermine levels were positively correlated with the patients' cardiac outputs (COs) ( $r=0.54, P=0.03$ ) (Fig. 3A). And levels of (2e)-2,5-dichloro-4-oxo-2-hexenedioic acid were positively correlated with the patients' serum creatinine ( $\mathrm{Scr}$ ) levels $(r=0.51, P=0.03)$ (Fig. 3B). There was no relationship between other significantly differential metabolites and the pulmonary artery pressure or the mPAP.

\section{Values of metabolites in predicting prognosis of lung transplantation}

All PAH patients were followed up after lung transplantation. The postoperative survival rate of patients with higher thymine levels ( $\geqslant$ arithmetic mean) in lung tissues was notably higher than that of patients with lower thymine levels $(P=0.038)$ (Fig. 3C). However, in multivariate COX model, patients with high level of thymine did not show significant improvement in prognosis.

\section{AKT as a central pathway in PAH metabolomics}

We identified that 7 significantly differential metabolites were reported to be related to AKT pathways by literature reviewing. AKT pathway has been validated to be involved in the occurrence and development of PAH PASMCs and hypoxic rodent models $[17,18]$. The regulatory network of differential metabolites and AKT pathway was illustrated in Fig. 4A. In addition, protein expression levels of AKT and phosphorylation levels of AKT (Thr308) were decreased in both human PAH lungs (Fig. 4B, C) and MCTinduced PAH rat lungs (Fig. 4D, E). Total AKT protein expression could be induced by treating PASMCs with hypoxia in a time-dependent manner, reaching the maximal levels at $2 \mathrm{~h}(P<0.001$ compared with those in the control group), and decreased afterwards; while the phosphorylation levels of AKT were significantly increased by the first hour of hypoxia treatment and decreased after $24 \mathrm{~h}$ (Fig. 4F, G).

\section{Discussion}

In this study, a non-targeted LC-MS/MS-based metabolomics investigation was performed to identify metabolic features of PAH lungs. To the best of our knowledge, this is the first metabolomics study using explanted lungs from PAH patients, which, to a certain extent, excludes the possible heterogeneity of the in vitro models, rodent in vivo models or human peripheral blood samples.

As $\mathrm{PH}$ is divided into five categories, $\mathrm{iPAH}$ in the PAH category is one of the categories that may be closely related to genetic factors[19]. Lung transplantation, as a treatment for iPAH, is mostly performed 
in young patients. From the perspective of age matching, the adjacent tissues of young lung cancer patients should be selected. But considering that young lung cancer patients may have genetic specificity, even the lung tissues far from the tumor is not an ideal control. So we choose the non-tumor lung tissues of middle-aged and elderly patients with lung cancer as controls, which is considered acceptable in other PAH studies[20].

This study suggests that PAH patients do have significant differences in orthotopic lung tissue metabolism compared with control patients, and shares some similarities with previous in vitro and in vivo metabolomics reports.

In our study, thymine decreased markedly in both iPAH and CHD-PAH lungs. In previous researches [21, 22], the decreased expression of thymine is usually accompanied by high expression of thymidine phosphorylase (TP). TP upregulates the methylation level of IRF8, and thereby enhances the expression of nuclear factor of activated T cells cytoplasmic 1 protein (NFATC1). And increased NFATc1 is also a characteristic pathogenic feature in $\mathrm{PAH}$ development, which decreases the expression of mitochondrial enzymes and members of the Bcl-2 family [23]. In addition, iPAH patients' thymine levels are significantly lower than that of controls, but higher than that of CHD-PAH patients. And the multi-factor prognostic analysis suggests that patients with higher thymine expression has significantly better prognosis than those with low expression after lung transplantation [24]. Thus, we speculate that thymine could be a biomarker of the prognosis of $\mathrm{PAH}$ patients receiving lung transplantation.

Neopterin (NP), which is able to interfere with reactive species and then promotes oxidative stress, decreases in all PAH lungs in this study. As well established, the dysregulation of the nitricoxide (NO) pathway works as a key element of PAH etiobiology. The interaction of NP with the intermediates and its ability to amplify the effects of various reactive oxygen species may be important for the progression of PAH $[25,26]$. NP affects vital molecule functions in the development of PAH, such as the AKT phosphorylation, and leads to the final end of PAH [27]. Thus, we can infer that the lack of endogenous or exogenous specific metabolic substances is the cause of PAH development.

In addition, the changes of some metabolites are inconsistent with previous reports. For example, the content of taurine increases in the lung tissues of hypoxic rodent models [28], which is contrary to our results. It is found that oral taurine administration attenuates vascular remodeling in hypoxic rat lungs, whereas depletion of endogenous taurine by administration of beta-alanine results in increased vascular remodeling [29]. The role of endogenous taurine supports our results. Meanwhile, the concentrations of some other metabolites, which have not been reported to be related to PAH, such as n-acetyl-I-aspartic acid and (2e)-2,5-dichloro-4-oxo-2-hexenedioic acid, change significantly in PAH.

Moreover, valuable metabolic pathways were identified in PAH. By exploring the inner connection of these metabolic pathways, 26 metabolic pathways associated with AKT pathways are found. AKT is activated by a number of receptor tyrosine kinases following the binding of growth factors or hormones such as PDGF and insulin, working as an important mediator of the 3-phosphoinositide-dependent kinase (PI3K) pathway [30-33]. Activated AKT can increase the expression of many pro-proliferative genes such as Bcl- 
2, decrease pro-apoptotic genes ( $\mathrm{Kv}$ channels), and then lead to PAH [34]. However, in our results, phosphorylation levels of AKT decrease in PAH patients. Among the 7 differential metabolites in the metabolic pathway, spermidine, spermine and glycine decrease but taurine increases in PAH. Despite the contrary results, their effects on phosphorylated AKT are in line with previous reports that increased concentrations of spermidine, spermine and decreased concentration of glycine can inhibit the phosphorylation of AKT $[32,33,35]$. In order to verify the activation of the AKT pathway in PAH, we performed gradient hypoxia on PASMCs and found that phosphorylation levels of AKT increased during short-term hypoxia, but then decreased comparing with the untreated sample. This result suggests the dynamic activation of AKT pathway that increasing in the early onset of PAH but decreasing afterwards.

This study also has a few shortcomings. First, our samples are quite limited due to the low proportion of $\mathrm{PAH}$ patients receiving lung transplantation. Second, due to ethical and objective factors, the lung tissues far from the tumor are taken as controls instead of lungs from healthy individuals.

\section{Conclusions}

Significant metabolic features of PAH are identified from explanted lungs. Specifically, differential metabolites may affect the PAH process by affecting the biological function of AKT in different courses of the disease, suggesting that AKT can be used as a potential target for PAH treatment. And our results update the understanding of the mechanisms of $\mathrm{PAH}$ pathogenesis and the improvement of therapeutic strategies targeting metabolic alterations in PAH.

\section{Abbreviations}

\section{PAH}

Pulmonary arterial hypertension

\section{PLS-DA}

Partial least squares discriminant analysis

\section{HPASMCs}

Human pulmonary artery smooth muscle cells

\section{cos}

Cardiac outputs

Scr

Serum creatinine

$\mathrm{PH}$

Pulmonary hypertension

mPAP

mean pulmonary arterial pressure

PAWP

Pulmonary artery wedge pressure

PVR 
Pulmonary vessel resistance

WU

Wood Units

WSPH

World Symposium on Pulmonary Hypertension

iPAH

idiopathic $\mathrm{PAH}$

CHD-PAH

$\mathrm{PAH}$ associated with congenital heart disease

LC-MS/MS

Liquid chromatography-tandem mass spectrometry

COPD

Chronic obstructive pulmonary disease

PFA

Paraformaldehyde

H\&E

Hematoxylin and eosin

media/CSA

Medial thickness to cross sectional area

QC

Quality control

IT

Injection time

NCE

Normalized collision energies

PQN

Probabilistic quotient normalization

QC-RLSC

Quality control-based robust LOESS signal correction

CVs

Coefficient of variations

VIP

Variable important for the projection

MCT

Monocrotaline

FBS

Fetal bovine serum

BMI

Body mass index

PA 
Pulmonary artery

FC

Functional capacity

EF

Ejection fractions

TP

Thymidine phosphorylase

NFATc1

Nuclear factor of activated T cells cytoplasmic 1 protein

NP

Neopterin

NO

Nitricoxide

PI3K

3-phosphoinositide-dependent kinase

\section{Declarations}

\section{Ethics approval and consent to participate}

All participants in this study agreed to donate their cut-off tissues for research. Written informed consents were obtained from all patients in accordance with the declaration of Helsinki. This study was approved by the Nanjing Medical University Ethics Committee ([2015] No.35, China).

Animal study was approved by the Nanjing Medical University Ethics Committee (IACUC-2105005, China). The animals were maintained in a constant environmental condition (temperature $23 \pm 2^{\circ} \mathrm{C}$, humidity $55 \pm$ $5 \%, 12: 12 \mathrm{~h}$ light/dark cycle) in the Animal Research Center of Nanjing Medical University. They had free access to food and water before and after all procedures.

\section{Consent for publication}

Not applicable.

\section{Availability of data and materials}

Due to protection of patient data privacy, sharing of data on a publicly available repository is not possible, but data are available from the corresponding authors upon request.

\section{Competing interests}

All authors report no conflict of interest

\section{Funding}


This research was supported by National Natural Science Foundation of China (81772020, 82002028) and Medical and Technology Development Program of Nanjing (YKK17115).

\section{Contributions}

Jingjing Ding: conceptualization, methodology, acquisition of data, analysis and interpretation of data, writing (original draft). Chunyan Chu: investigation, acquisition of data, analysis and interpretation of data, writing (review and editing). Zhengsheng Mao: conceptualization, methodology. Jiawen Yang: analysis and interpretation of data. Jie Wang: validation. Li Hu: acquisition of data. Peng Chen: acquisition of data. Yue Cao: analysis and interpretation of data. Yan Li: analysis and interpretation of data. Hua Wan: methodology. Dong Wei: methodology. Jingyu Chen: conceptualization, methodology, acquisition of data. Feng Chen conceptualization, methodology, acquisition of data. Youjia Yu: conceptualization, methodology, acquisition of data, analysis and interpretation of data, validation, writing. All authors read and approved the final manuscript.

\section{Acknowledgements}

Not applicable.

\section{References}

1. Sommer N, Ghofrani HA, Pak O, Bonnet S, Provencher S, Sitbon O, et al. Current and future treatments of pulmonary arterial hypertension. Br J Pharmacol. 2021;178(1):6-30.

2. Hoeper MM, Humbert M, Souza R, Idrees M, Kawut SM, Sliwa-Hahnle K, et al. A global view of pulmonary hypertension. Lancet Respir Med. 2016;4(4):306-22.

3. Simonneau G, Montani D, Celermajer DS, Denton CP, Gatzoulis MA, Krowka M, et al. Haemodynamic definitions and updated clinical classification of pulmonary hypertension. Eur Respir J. 2019;53(1).

4. Lau EMT, Giannoulatou E, Celermajer DS, Humbert M. Epidemiology and treatment of pulmonary arterial hypertension. Nat Rev Cardiol. 2017;14(10):603-14.

5. Lim Y, Low TT, Chan SP, Teo TW, Jang JJ, Yip N, et al. Pulmonary arterial hypertension in a multiethnic Asian population: Characteristics, survival and mortality predictors from a 14-year follow-up study. Respirology. 2019;24(2):162-70.

6. Fernandes CJC, Jardim C, Souza R. The global view. Curr Opin Pulm Med. 2019;25(5):391-7.

7. Galie N, Humbert M, Vachiery JL, Gibbs S, Lang I, Torbicki A, et al. 2015 ESC/ERS Guidelines for the diagnosis and treatment of pulmonary hypertension: The Joint Task Force for the Diagnosis and Treatment of Pulmonary Hypertension of the European Society of Cardiology (ESC) and the European Respiratory Society (ERS): Endorsed by: Association for European Paediatric and Congenital Cardiology (AEPC), International Society for Heart and Lung Transplantation (ISHLT). Eur Heart J. 2016;37(1):67-119. 
8. Sikirica M, lorga SR, Bancroft T, Potash J. The economic burden of pulmonary arterial hypertension (PAH) in the US on payers and patients. BMC Health Serv Res. 2014;14:676.

9. Harbaum L, Rhodes CJ, Otero-Nunez P, Wharton J, Wilkins MR. The application of 'omics' to pulmonary arterial hypertension. Br J Pharmacol. 2021;178(1):108-20.

10. Hoeper MM, Huscher D, Ghofrani HA, Delcroix M, Distler O, Schweiger C, et al. Elderly patients diagnosed with idiopathic pulmonary arterial hypertension: results from the COMPERA registry. Int $\mathrm{J}$ Cardiol. 2013;168(2):871-80.

11. Boucherat O, Peterlini T, Bourgeois A, Nadeau V, Breuils-Bonnet S, Boilet-Molez S, et al. Mitochondrial HSP90 Accumulation Promotes Vascular Remodeling in Pulmonary Arterial Hypertension. Am J Respir Crit Care Med. 2018;198(1):90-103.

12. Milisav I, Ribaric S, Poljsak B. Antioxidant Vitamins and Ageing. Subcell Biochem. 2018;90:1-23.

13. Reitz BA, Wallwork JL, Hunt SA, Pennock JL, Billingham ME, Oyer PE, et al. Heart-lung transplantation: successful therapy for patients with pulmonary vascular disease. $\mathrm{N}$ Engl J Med. 1982;306(10):557-64.

14. Sanders JL, Han Y, Urbina MF, Systrom DM, Waxman AB. Metabolomics of exercise pulmonary hypertension are intermediate between controls and patients with pulmonary arterial hypertension. Pulm Circ. 2019;9(4):2045894019882623.

15. Chen C, Luo F, Wu P, Huang Y, Das A, Chen S, et al. Metabolomics reveals metabolite changes of patients with pulmonary arterial hypertension in China. J Cell Mol Med. 2020;24(4):2484-96.

16. Chen XJ, Li K, Xu L, Yu YJ, Wu B, He YL, et al. Novel insight from the first lung transplant of a COVID19 patient. Eur J Clin Invest. 2021;51(1):e13443.

17. Li Y, Yang L, Dong L, Yang ZW, Zhang J, Zhang SL, et al. Crosstalk between the Akt/mTORC1 and NFkappaB signaling pathways promotes hypoxia-induced pulmonary hypertension by increasing DPP4 expression in PASMCs. Acta Pharmacol Sin. 2019;40(10):1322-33.

18. Di R, Yang Z, Xu P, Xu Y. Silencing PDK1 limits hypoxia-induced pulmonary arterial hypertension in mice via the Akt/p70S6K signaling pathway. Exp Ther Med. 2019;18(1):699-704.

19. Zeng H, Liu X, Zhang Y. Identification of Potential Biomarkers and Immune Infiltration Characteristics in Idiopathic Pulmonary Arterial Hypertension Using Bioinformatics Analysis. Front Cardiovasc Med. 2021;8:624714.

20. Rhodes CJ, Ghataorhe P, Wharton J, Rue-Albrecht KC, Hadinnapola C, Watson G, et al. Plasma Metabolomics Implicates Modified Transfer RNAs and Altered Bioenergetics in the Outcomes of Pulmonary Arterial Hypertension. Circulation. 2017;135(5):460-75.

21. Lee SJ, Yeo JS, Lee HJ, Lee EJ, Kim SY, Jang SJ, et al. Thymidine phosphorylase influences [(18)F]fluorothymidine uptake in cancer cells and patients with non-small cell lung cancer. Eur $\mathrm{J}$ Nucl Med Mol Imaging. 2014;41(7):1327-35.

22. Schardt C, Rotsch M, Erbil C, Goke R, Richter G, Havemann K. Characterization of insulin-like growth factor II receptors in human small cell lung cancer cell lines. Exp Cell Res. 1993;204(1):22-9. 
23. Paulin R, Michelakis ED. The metabolic theory of pulmonary arterial hypertension. Circ Res. 2014;115(1):148-64.

24. Liu H, Liu Z, Du J, He J, Lin P, Amini B, et al. Thymidine phosphorylase exerts complex effects on bone resorption and formation in myeloma. Sci Transl Med. 2016;8(353):353ra113.

25. Widner B, Baier-Bitterlich G, Wede I, Wirleitner B, Fuchs D. Neopterin derivatives modulate the nitration of tyrosine by peroxynitrite. Biochem Biophys Res Commun. 1998;248(2):341-6.

26. Weiss G, Fuchs D, Hausen A, Reibnegger G, Werner ER, Werner-Felmayer G, et al. Neopterin modulates toxicity mediated by reactive oxygen and chloride species. FEBS Lett. 1993;321(1):89-92.

27. Smukowska-Gorynia A, Marcinkowska J, Chmara E, Malaczynska-Rajpold K, Slawek-Szmyt S, Cieslewicz A, et al. Neopterin as a Biomarker in Patients with Pulmonary Arterial Hypertension and Chronic Thromboembolic Pulmonary Hypertension. Respiration. 2018;96(3):222-30.

28. Li M, Riddle S, Zhang H, D'Alessandro A, Flockton A, Serkova NJ, et al. Metabolic Reprogramming Regulates the Proliferative and Inflammatory Phenotype of Adventitial Fibroblasts in Pulmonary Hypertension Through the Transcriptional Corepressor C-Terminal Binding Protein-1. Circulation. 2016;134(15):1105-21.

29. Shiota M, Kusakabe H, Hikita Y, Nakao T, Izumi Y, Iwao H. Pharmacogenomics of cardiovascular pharmacology: molecular network analysis in pleiotropic effects of statin -- an experimental elucidation of the pharmacologic action from protein-protein interaction analysis. J Pharmacol Sci. 2008;107(1):15-9.

30. Yang H, Lee SE, Kim GD, Park HR, Park YS. Hemeoxygenase-1 mediates an adaptive response to spermidine-induced cell death in human endothelial cells. Oxid Med Cell Longev. 2013;2013:238734.

31. Rajeeve V, Pearce W, Cascante M, Vanhaesebroeck B, Cutillas PR. Polyamine production is downstream and upstream of oncogenic PI3K signalling and contributes to tumour cell growth. Biochem J. 2013;450(3):619-28.

32. Wei C, Li HZ, Wang YH, Peng X, Shao HJ, Li HX, et al. Exogenous spermine inhibits the proliferation of human pulmonary artery smooth muscle cells caused by chemically-induced hypoxia via the suppression of the ERK1/2- and PI3K/AKT-associated pathways. Int J Mol Med. 2016;37(1):39-46.

33. Kim B, Arany Z. Does. Glycine cataBOLAsm Drive Pulmonary Hypertension? Circulation. 2019;139(19):2256-9.

34. Dumas de la Roque E, Savineau JP, Bonnet S. Dehydroepiandrosterone. A new treatment for vascular remodeling diseases including pulmonary arterial hypertension. Pharmacol Ther. 2010;126(2):18699.

35. Izquierdo-Garcia JL, Arias T, Rojas Y, Garcia-Ruiz V, Santos A, Martin-Puig S, et al. Metabolic Reprogramming in the Heart and Lung in a Murine Model of Pulmonary Arterial Hypertension. Front Cardiovasc Med. 2018;5:110.

\section{Figures}


A

Con

\section{$\mathrm{PAH}$}
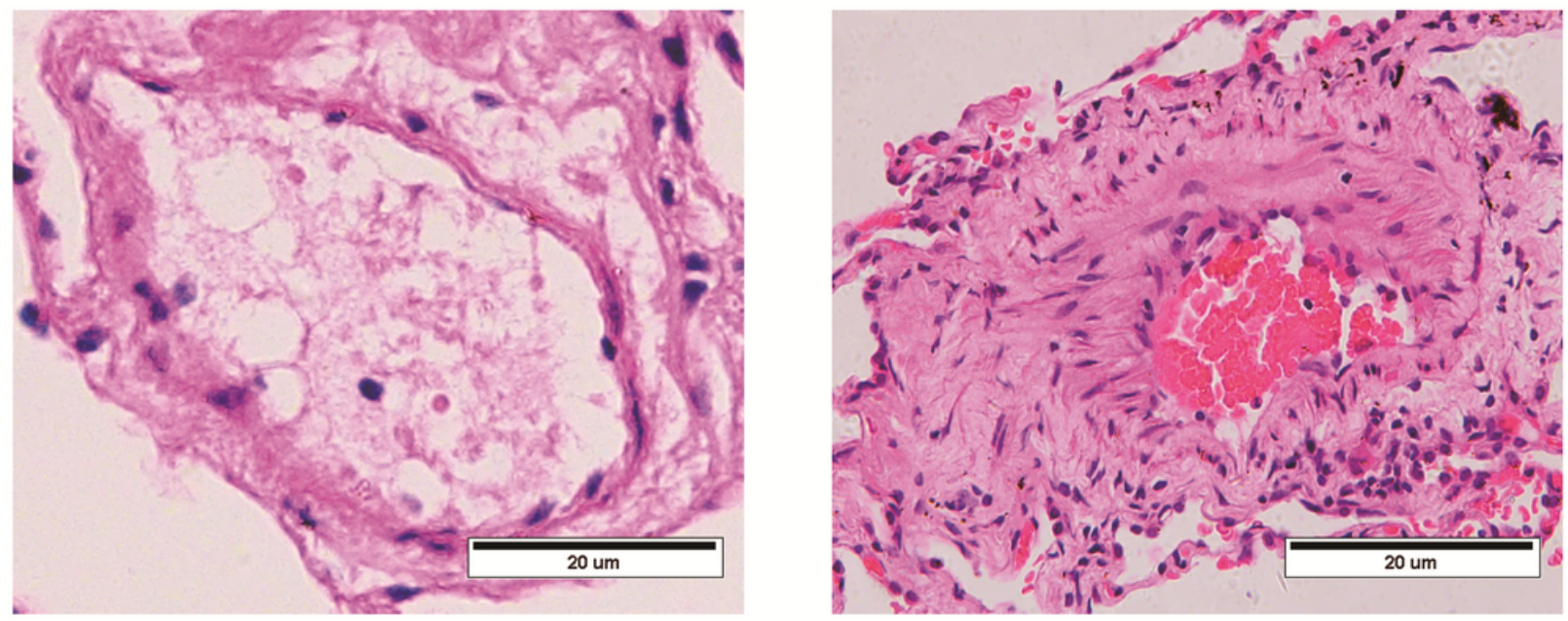

B

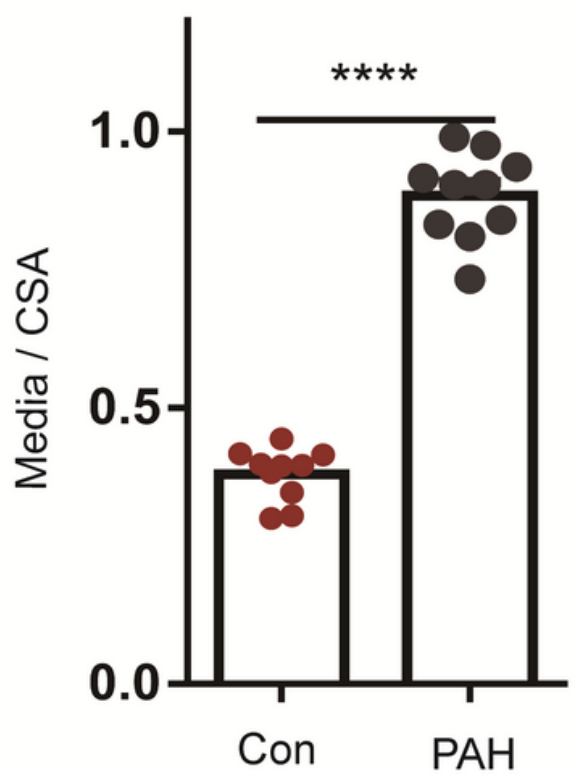

C

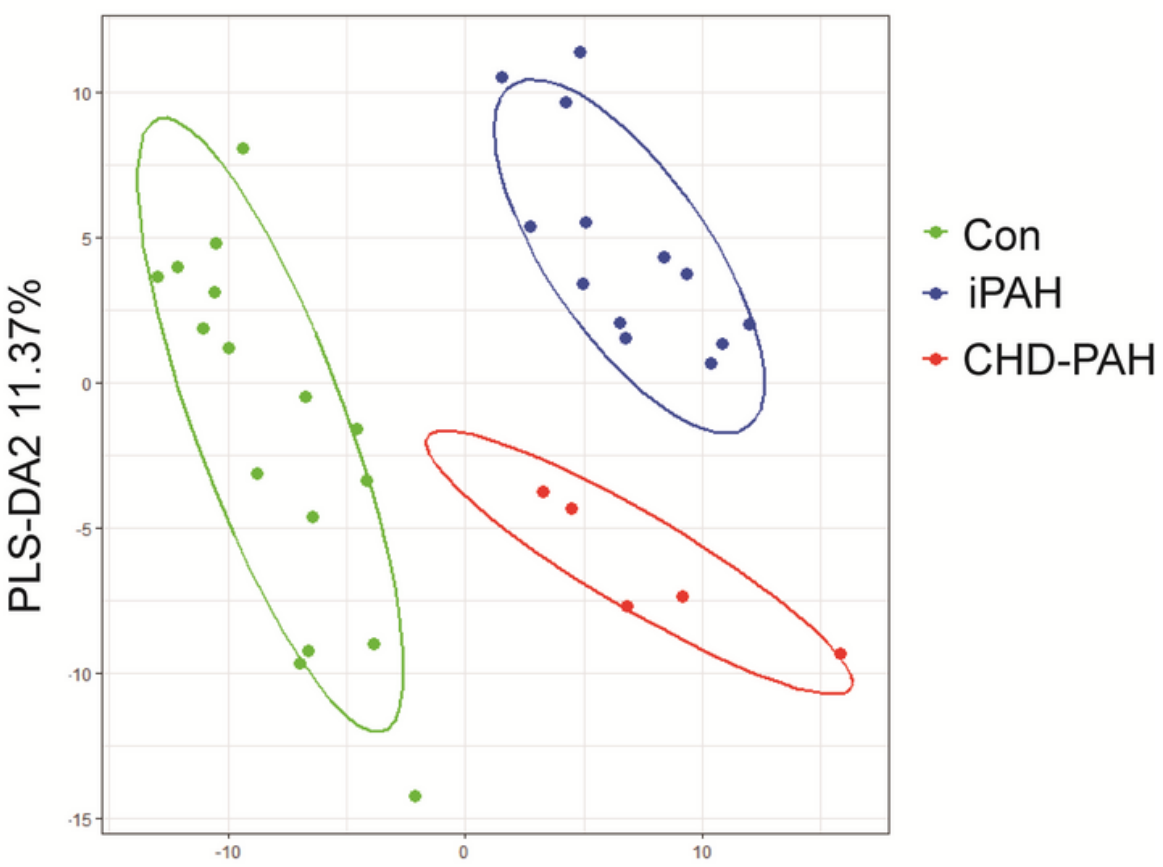

PLS-DA1 $14.94 \%$

\section{Figure 1}

The histopathological characteristics and metabolomics diversity of PAH and control lung tissues. (A) characteristic pulmonary arteries in PAH and control lung tissues (Scale bar, $50 \mu \mathrm{m}$ ). (B) 10 muscular arteries with diameters ranging from $20 \mu \mathrm{m}$ to $100 \mu \mathrm{m}$ were randomly selected from the lung slides of PAH and control. Quantification data were shown as the ratio of vascular medial thickness to total vessel size (media/cross-sectional area (CSA)), ${ }^{\star \star * * P}<0.0001$. (C) Score graph of Partial least squares discriminant analysis (PLS-DA) shows IPAH (blue) and CHD-PAH (red) as compared with the control (green) in combined ion model. Con, the control. 
A

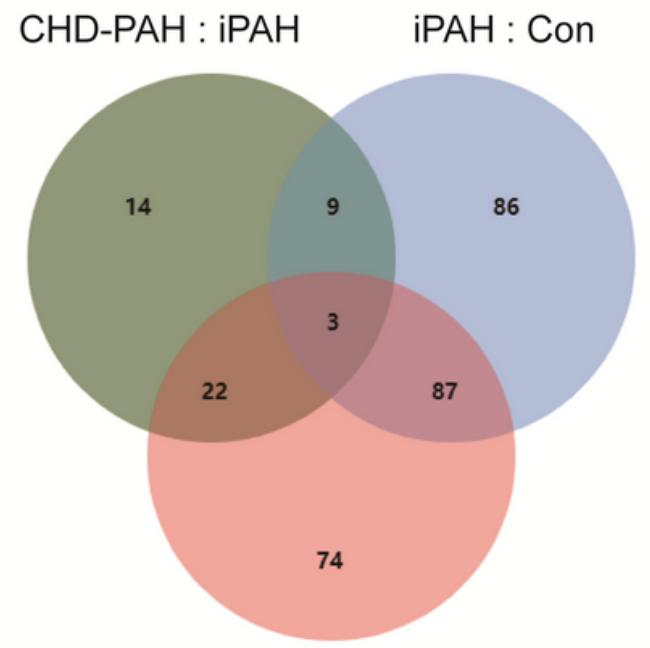

CHD-PAH : Con
B

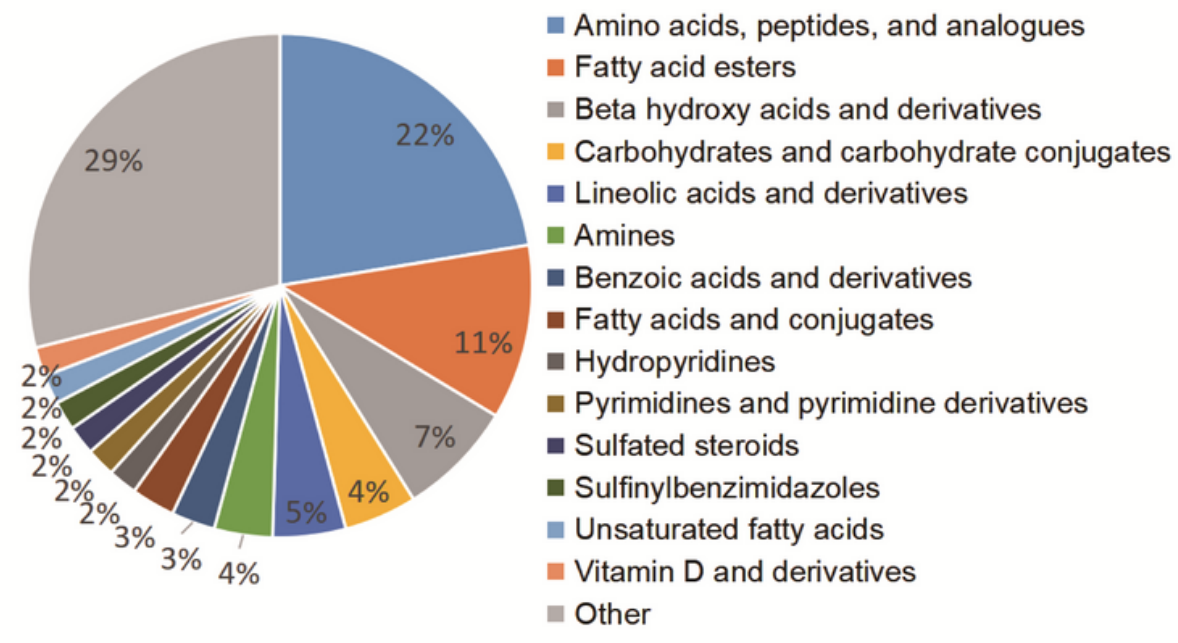

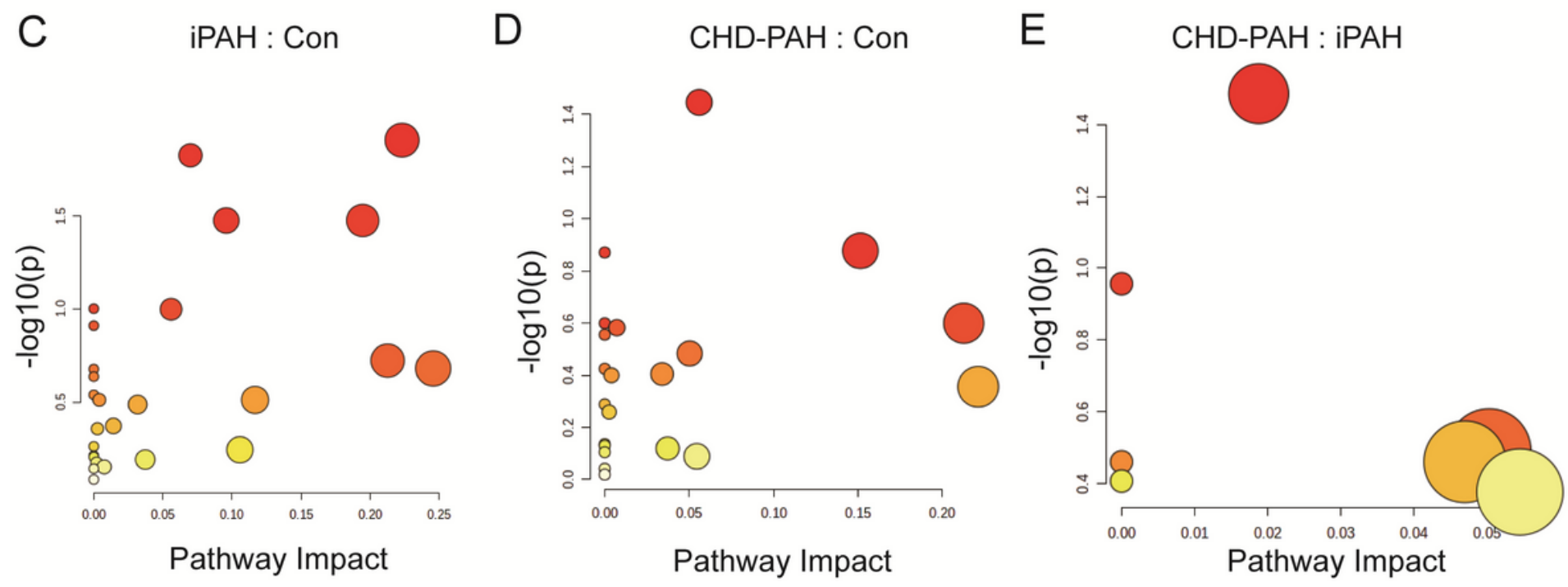

Figure 2

The metabolite alterations and metabolic pathway changes among iPAH, CHD-PAH and control lungs. (A) The Venn diagram showed the significantly changed metabolites in iPAH vs. control, CHD-PAH vs. control and iPAH vs. CHD-PAH comparisons. (B) The pie chart indicated the percentage of each metabolic pathway in total metabolic pathways that obtained from pathway enrichment analysis based on the KEGG database. The graphical output of summary of pathway analysis by MetaboAnalyst 5.0 in (C) iPAH vs. control, (D) CHD-PAH vs. control and (E) iPAH vs. CHD-PAH. Con, the control. 


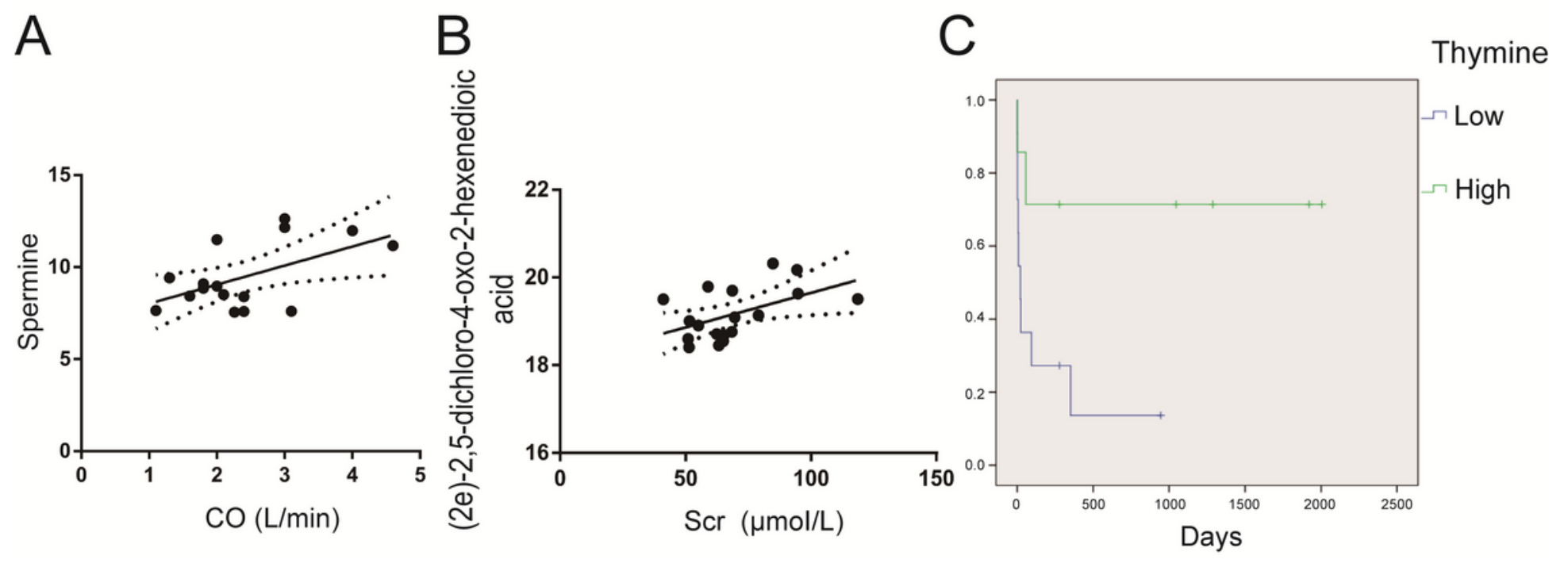

Figure 3

The metabolite alterations and metabolic pathway changes among iPAH, CHD-PAH and control lungs. (A) The Venn diagram showed the significantly changed metabolites in iPAH vs. control, CHD-PAH vs. control and IPAH vs. CHD-PAH comparisons. (B) The pie chart indicated the percentage of each metabolic pathway in total metabolic pathways that obtained from pathway enrichment analysis based on the KEGG database. The graphical output of summary of pathway analysis by MetaboAnalyst 5.0 in (C) iPAH vs. control, (D) CHD-PAH vs. control and (E) iPAH vs. CHD-PAH. Con, the control. 
A

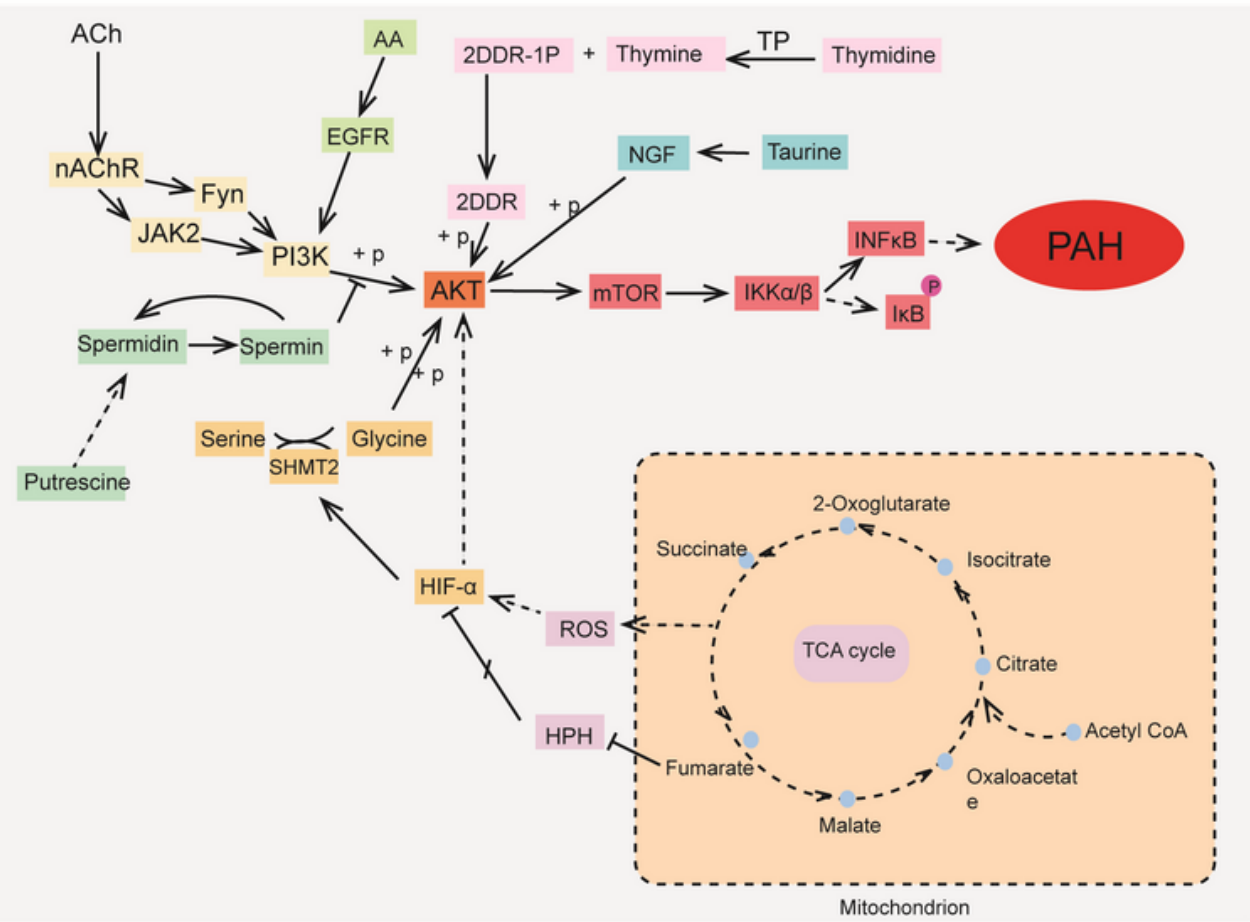

B

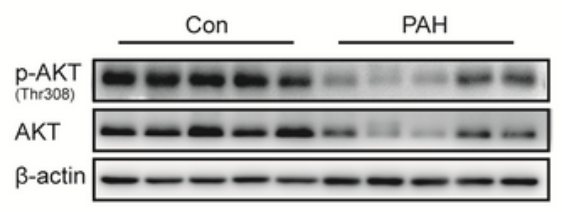

C

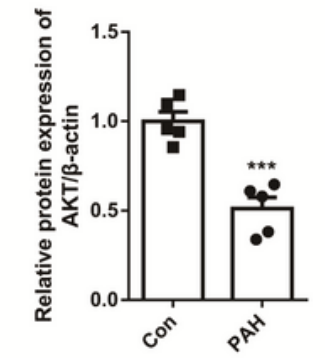

$\mathrm{F}$

$\begin{array}{lllllllll}\text { Hypoxia time (h) } & 0 & 0.5 & 1 & 2 & 4 & 8 & 16 & 24\end{array}$

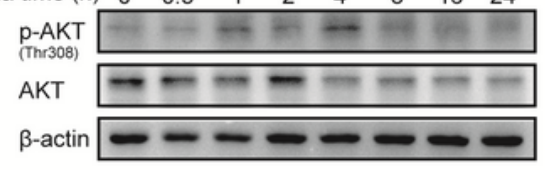

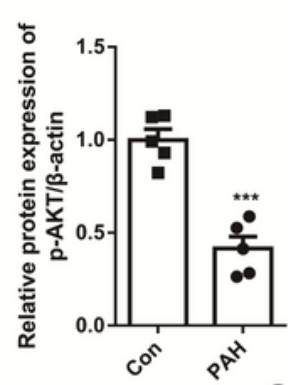

G
D

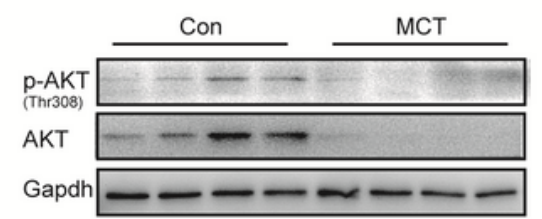

E

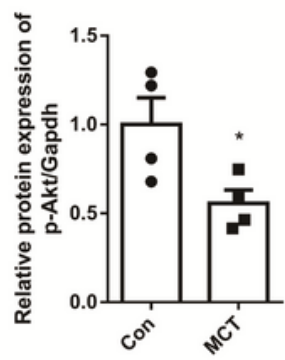

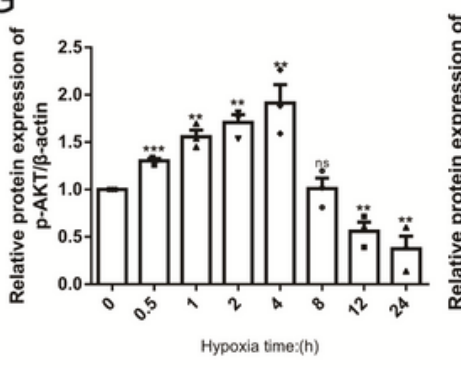
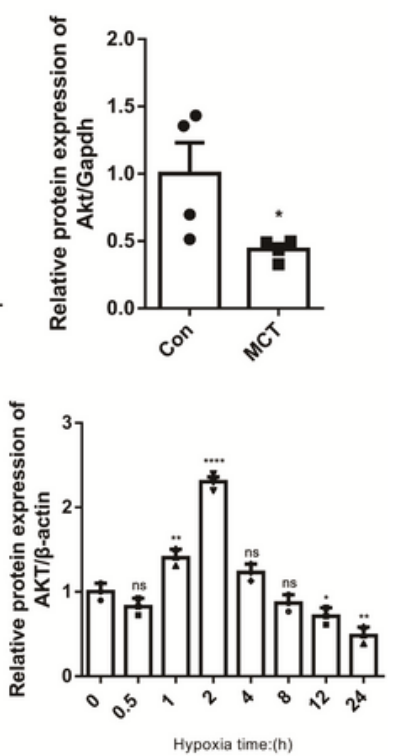

\section{Figure 4}

AKT as a central pathway in PAH metabolomics. (A) The possible mechanism of differential metabolites in process of PAH. Different colors represent different metabolites and related molecules. (B, C) Protein expression of indicated proteins were determined by Western blotting in control and PAH lungs $(n=5 /$ group), (D, E) control and MCT-induced PAH rat lungs ( $n=4$ / group) and $(F, G)$ PASMCs with different durations of hypoxia treatment. All statistical data were from three independent experiments. In all 


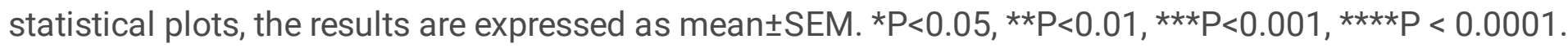
n.s., not significant, by two-tailed Student's t test. Source data are provided as a Source Data file.

\section{Supplementary Files}

This is a list of supplementary files associated with this preprint. Click to download.

- Supplementarymaterial.docx 